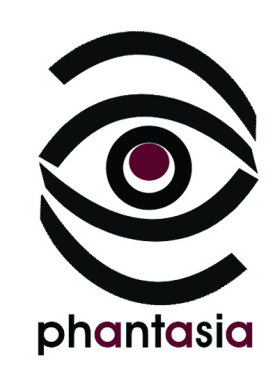

\title{
Une fidélité de Foucault à Nietzsche : le langage comme fil conducteur généalogique
}

\author{
Emmanuel Salanskis \\ Université de Strasbourg
}

L'objet de cet article est d'examiner la relation entre Nietzsche et Foucault sous un angle particulier, qui est le rôle du langage en tant que fil conducteur possible d'une investigation généalogique. Mon propos portera par conséquent sur une force des mots qui se révélerait spécifiquement au généalogiste. À cet égard, il existe à mon avis une filiation intéressante entre Foucault et Nietzsche, qu'on peut même comprendre comme une fidélité silencieuse du premier au second.

Ayant prononcé ce mot de «fidélité », je dois immédiatement envisager une difficulté. Il semblerait que Foucault ait exclu par avance ce genre de commentaire. Dans un «Entretien sur la prison » publié en 1975, où il souligne lui-même la présence de Nietzsche dans ses recherches, Foucault déclare pourtant à ce propos : "Que les commentateurs disent si l'on est ou non fidèle, cela n'a aucun intérêt " ${ }^{1}$. Cette déclaration semble péremptoire, mais elle doit en réalité être interprétée avec précaution, car tout dépend du sens qu'on donne au mot «fidélité ». On peut ainsi distinguer une fidélité qui s'exprime dans l'exactitude du commentaire et une fidélité qui se traduit par la reprise d'une démarche. C'est la première des deux qui peut effectivement apparaître comme secondaire dans le cas de Foucault : il dira lui-même en 1984 qu'il n'a écrit sur Nietzsche " qu'un tout petit article », ce qui est sans doute exagéré, mais néanmoins révélateur d'une intention, c'est-àdire d'une remarquable volonté de silence sur un auteur qui lui était si proche ${ }^{2}$. Pour autant, au-delà de l'exégèse explicite, Foucault s'est aussi efforcé d'utiliser, de déformer, de faire grincer et même de faire crier la pensée nietzschéenne : c'est ce qu'il souligne précisément dans le même entretien de $1975^{3}$. Si on veut comprendre l'inspiration nietzschéenne de Foucault, qui est une appropriation créatrice, il faut par conséquent considérer son propre travail de généalogiste, même quand Nietzsche n'y est pas expressément nommé. C'est l'approche que je privilégierai dans cet article. Je voudrais essayer de montrer que le Foucault généalogiste des cours au Collège de France a pris en compte, dans sa propre méthode, un aspect précis et crucial de l'enquête menée par Nietzsche dans la Généalogie de la morale. Ce passage de témoin est à mes yeux une des principales raisons qui autorisent à parler de la généalogie comme d'une tradition philosophique à part entière ${ }^{4}$. Mais je ne vais proposer à ce sujet qu'une perspective liminaire et exploratoire : je sollicite donc l'indulgence du lecteur, notamment foucaldien, en lui demandant de ne pas me reprocher l'absence d'exhaustivité de cette première tentative.

\footnotetext{
${ }^{1}$ Cf. Foucault (M.), « Entretien sur la prison : le livre et sa méthode », entretien avec J.- J. Brochier, Magazine littéraire, $\mathrm{n}^{\circ}$ 101, juin 1975, p. 27-33 (repris dans Dits et écrits : 1954-1988, op. cit., vol. 2, p. 740-753, ici p. 753).

${ }^{2}$ Cf. Foucault (M.), « Le retour de la morale », entretien avec G. Barbedette et A. Scala, 29 mai 1984, Les Nouvelles Littéraires, $\mathrm{n}^{\circ}$ 2937, 28 juin-5 juillet 1984 (repris dans Dits et écrits : 1954-1988, sous la direction de D. Defert et Fr. Ewald, Paris, Gallimard, 1994, vol. 4, p. 696-707, ici p. 703). Cf. aussi FouCAUlT (M.), « Nietzsche, la généalogie, 1'histoire », Hommage à Jean Hyppolite, Paris, PUF, 1971, p. 145-172, repris dans Dits et écrits : 1954-1988, op. cit., vol. 2, p. 136-156.

${ }^{3}$ Cf. Foucault (M.), « Entretien sur la prison : le livre et sa méthode », op. cit., p. 753.

${ }^{4}$ Sur l'idée que la généalogie constitue une tradition philosophique, cf. KoOPMAN (C.), Genealogy as Critique. Foucault and the Problems of Modernity, Bloomington/Indianapolis, Indiana University Press, 2013.
} 
Cette précision donnée, j'en viens à l'aspect généalogico-langagier qui est au cœur de la transmission qui m'intéresse. Pour mettre cet aspect en évidence chez Nietzsche, je vais m'appuyer sur une indication de méthode qui est fournie par le premier traité de la Généalogie de la morale, d'abord au $\S 4$ puis au $§ 17$, donc quasiment au début et ensuite à l'extrême fin du traité. En donnant cette indication deux fois et dans deux emplacements particulièrement significatifs, Nietzsche inscrit globalement le premier traité dans un même cadre méthodologique, qu'il revendique d'ailleurs comme son originalité, pour se démarquer d'auteurs qu'il appelle les «psychologues anglais » ou les « généalogistes de la morale jusqu'ici ». Dans la première occurrence, Nietzsche souligne ce qui l'a mis sur la bonne voie en matière de généalogie de la morale à la différence d'auteurs comme Paul Rée et Herbert Spencer : "L'indication révélant la voie correcte me fut donnée par cette question, que peuvent bien signifier, au point de vue étymologique, les désignations du "bon" forgées dans les différentes langues ? ${ }^{6}$. Nietzsche préconise donc une "voie correcte » qui consiste à suivre un fil conducteur langagier. Il s'agit bien sûr d'une traduction concrète du fameux thème du "gris des documents », qui avait été évoqué au $\S 7$ de la préface : si l'on veut étudier la "réelle histoire de la morale», au lieu de se perdre dans l'azur des "constructions hypothétiques anglaises ", il faut déchiffrer des documents tangibles, qui nous renseigneront en premier lieu sur l'histoire du vocabulaire moral. Cette indication de méthode est suffisamment importante pour être réaffirmée par la « note » qui conclut le premier traité. Exprimant son intention de réorienter les études d'histoire de la morale, Nietzsche demande en particulier aux facultés de philosophie de primer des mémoires qui porteraient sur la question suivante: "Quelles sont les indications que fournit la linguistique, et notamment la recherche étymologique, relativement à l'histoire du développement des concepts moraux? ». Dans cette note conclusive, Nietzsche pose donc une question langagière et étymologique, qui pourrait sembler modeste aux adeptes de la pensée conceptuelle, mais qui mérite selon lui «l'attention des philologues et des historiens tout autant que celle des universitaires spécialisés par profession en philosophie ».

Pourquoi présenter l'histoire du langage comme un enjeu si crucial pour le généalogiste de la morale ? Mon but sera de justifier cette exigence méthodologique formulée par Nietzsche, tout en montrant que Foucault l'a prise au sérieux dans son propre travail. J'annonce le plan en deux parties que je vais suivre. La question langagière de Nietzsche recouvre à mon avis deux enjeux, qui correspondent chacun à une certaine " force des mots ». Mais cette force serait illusoire dans un cas et heuristique dans l'autre. Du premier point de vue, revenir au vocabulaire moral réel dans sa pluralité et son devenir, c'est refuser l'interprétation essentialiste qui s'est attachée au mot " morale ». "Chaque mot est un préjugé », disait déjà un aphorisme du Voyageur et son ombre ${ }^{7}$. Et Par-delà bien et mal confirmera par la suite que la séduction du mot «morale » ou des mots « bien et mal » a entretenu les philosophes dans une ignorance grossière des faits : «ils étaient mal informés au sujet des peuples, des époques, des temps passés, et même peu curieux de les connaître $»^{8}$. Une première motivation pour en revenir à l'histoire des mots serait donc négative. Le langage ne doit surtout pas guider le généalogiste au sens où le prestige d'un mot unique conduirait à postuler un universel sous-jacent, comme si l'histoire n'était que le développement linéaire d'un seul et même concept. Foucault, on le sait, appellera cette illusion le « déploiement métahistorique des significations idéales et des indéfinies téléologies ${ }^{9}$. Mais justement, si cet obstacle est dûment repéré, il peut au contraire devenir un point d'appui pour l'enquête généalogique, car si on part du principe que les mots n'ont pas de signification intemporelle, on découvre à l'inverse leur pouvoir heuristique en tant que traces d'une histoire interprétative. Et c'est ce rôle plus positif du langage qui me paraît décisif à la fois chez Nietzsche et chez Foucault, ainsi que je le ferai valoir en deuxième partie.

\section{Le préjugé du mot}

Je commence donc par considérer l'aspect négatif du problème, c'est-à-dire le préjugé du mot en tant qu'il bride l'exercice de la généalogie. Comme je viens de le rappeler, Nietzsche dénonce ce travers chez ses

\footnotetext{
${ }^{5}$ Cf. NietzSChe (F.), Éléments pour la généalogie de la morale (désormais GM), Paris, Librairie Générale Française, 2000 , trad. P. Wotling, respectivement I, $\S 1$ et II, $\S 12$. Je modifie très légèrement la traduction de « die bisherigen Moral-Genealogen » proposée par Patrick Wotling.

${ }^{6}$ Cf. GM, I, § 4.

${ }^{7}$ Cf. NiETZSCHE (F.), Le Voyageur et son ombre, dans Humain, trop humain : un livre pour esprits libres, t. 2 , trad. R. Rovini revue par M. de Launey, Paris, Gallimard, 1991, § 55.

${ }^{8}$ Cf. Nietzsche (F.), Par-delà bien et mal, trad. P. Wotling, Paris, Flammarion, 2000, $§ 186$.

${ }^{9}$ Cf. Foucault M., « Nietzsche, la généalogie, l'histoire », Dits et écrits : 1954-1988, op. cit., vol. 2, p. 136.
} 
prédécesseurs immédiats, en particulier ceux qui se sont réclamés de la théorie de l'évolution. Le propre de ces auteurs était de construire des généalogies linéaires dans lesquelles une morale unique était présupposée, comme si l'émergence de cette morale ne pouvait s'expliquer que par une utilité sociale objective ayant existé de tout temps et sous le même rapport. Nietzsche observe que le support du mot joue un rôle essentiel dans ces reconstructions, parce qu'il fournit un point d'appui invariant qui donne l'illusion de parler d'une réalité unique tout au long du processus historique examiné.

L'illustration classique de cette méprise est la démarche de Paul Rée dans le petit livre sur L'Origine des sentiments moraux qu'il publie en $1877^{10}$. Tâchons toutefois de bien comprendre ce qui pose problème à Nietzsche dans l'analyse de Rée. Le défaut principal de l'ouvrage n'est pas, fondamentalement, de se référer à une anthropologie darwinienne. En réalité, avant même de convoquer la théorie de l'évolution, Rée a déjà commis une erreur beaucoup plus philosophique aux yeux de Nietzsche. Il croit savoir a priori ce que sont les sentiments moraux, et il le croit si bien qu'il règle la question en deux phrases : d'abord p. 1, avec l'affirmation d'un dualisme pulsionnel selon lequel « chaque homme réunit en lui deux pulsions, à savoir la pulsion égoïste et la pulsion non-égoïste ${ }^{11}$. Et ensuite, p. 9 , où la moralité est définie sur la base de ce dualisme, grâce au double postulat que «seules les actions égoïstes sont qualifiées de méchantes » et que «seules les actions non-égoïstes sont qualifiées de bonnes " ${ }^{12}$. Rée a donc posé un universel avant même d'amorcer son enquête historique, en admettant l'opposition judéo-chrétienne du bien et du mal, ou du bon altruisme et du méchant égoïsme. Cette démarche exclut d'emblée la possibilité que d'autres morales aient pu exister, au sens où l'altruisme n'aurait justement pas été universellement perçu comme le bien en soi : par exemple parce que les morales de l'honneur prescrivaient autrefois la vengeance, comme Nietzsche l'avait déjà remarqué au $\S 42$ d'Humain, trop humain; ou encore parce que des auteurs aussi différents que Platon, Spinoza, La Rochefoucauld ou Kant ont réprouvé la pitié, si on en croit le $\S 5$ de la préface de la Généalogie de la morale. D’où ce commentaire ironique de Nietzsche au $\S 7$ de la préface : le « Dr. Rée » ne connaissait pas « le long écrit hiéroglyphique [...] du passé de la morale humaine », " mais il avait lu Darwin - de sorte que dans ses hypothèses, de manière pour le moins divertissante, la bête darwinienne et le petit bout de chou de la morale "qui ne mord plus", suprêmement moderne et modeste, se tendent gentiment la main ${ }^{13}$. Nietzsche reproche par conséquent à Rée de darwiniser a posteriori une morale préalable. C'est ce présupposé moral qui permet d'écrire une généalogie linéaire de l'altruisme. Et ce parti pris repose à son tour sur une sélection arbitraire que Rée pratique, de fait, dans l'histoire du vocabulaire moral. Au premier traité de la Généalogie, Nietzsche répondra à Rée qu'il a oublié deux mots : l'opposition aristocratique entre gut et schlecht («bon et mauvais »), qui fonctionne selon une toute autre logique que l'opposition plébéienne entre gut et böse (« bon et méchant»).

Négativement, la généalogie nietzschéenne conduit donc à mettre en question l'universalité et l'invariance auxquelles le mot «morale » nous semble faussement renvoyer. Le singulier du titre allemand, Zur Genealogie der Moral, ne veut pas dire autre chose: c'est une généalogie de ce qu'on appelle communément "la morale », au singulier, sous l'effet d'un préjugé essentialiste et judéo-chrétien. Nietzsche ne souscrit évidemment pas à ce préjugé. Il est clair que de son point de vue, l'histoire ne donne jamais à voir que des morales, même si on peut classer ces modes d'évaluation en fonction de types récurrents, comme l'explique notamment le $\S 260$ de Par-delà bien et mal.

J'en arrive maintenant à la réception foucaldienne de cette critique du préjugé du mot. Il me semble que Foucault a retenu cet aspect négatif non seulement dans ses commentaires sur Nietzsche, mais aussi et surtout dans ses propres généalogies. Le premier point étant facile à mettre en évidence, je me contenterai de le mentionner rapidement. Dès la première page de «Nietzsche, la généalogie, l'histoire », Foucault écrit : «Paul Rée a tort, comme les Anglais, de décrire des genèses linéaires - d'ordonner, par exemple, au seul souci de l'utile, toute l'histoire de la morale ; comme si les mots avaient gardé leur sens [...] ${ }^{14}$. Je coupe ici la citation, car ce passage suffit à démontrer une réception du point langagier qui m'intéresse. Foucault met bien le doigt sur l'essentialisation du vocabulaire moral qui avait été critiquée par Nietzsche : contre cette

\footnotetext{
${ }^{10}$ Il faudrait également mentionner The Data of Ethics de Herbert Spencer, ce que je m'abstiens de faire ici dans un souci de concision. Sur la lecture nietzschéenne de Spencer, cf. SALANSKIS (E.), " Nietzsche lecteur de The Data of Ethics : une critique évolutionniste de la morale de Spencer », Arts et savoirs, $\mathrm{n}^{\circ} 4,2014$, en ligne, https://journals.openedition.org/aes/278.

${ }^{11}$ Cf. RÉE (P.), Der Ursprung der moralischen Empfindungen, Chemnitz, Verlag von Ernst Schmeitzner, 1877, p. 1, trad. ES.

${ }^{12}$ Ibid, trad. ES.

${ }^{13}$ Cf. GM, Préface, $\S 7$.

${ }^{14}$ Cf. Foucault (M.), « Nietzsche, la généalogie, l’histoire », op. cit., p. 136.
} 
essentialisation, il fait lui-même ressortir la question de la fluidité du sens des mots. Foucault a parfaitement compris cet enjeu et les pages dans lesquelles il l'exprime restent éclairantes aujourd'hui.

Il reste cependant à se demander ce que Foucault a fait de cette critique dans son propre travail généalogique. Sur ce point, je voudrais seulement ouvrir une perspective, sans prétendre le moins du monde livrer le genre d'analyse exhaustive qu'on attendrait dans une monographie. Je ne chercherai pas dans ce contexte à définir ce que Foucault a entendu par " généalogie »: même s'il est possible de caractériser la généalogie foucaldienne par un certain nombre d'indications que Foucault a données dans les années 1970 et 1980, une approche définitionnelle stricte me paraît en grande partie stérile, face à une méthode qui a ellemême évolué et qui ne s'est pas figée en doctrine ${ }^{15}$. Il y a d'ailleurs des raisons généalogiques fortes de refuser une telle approche définitionnelle, comme on le verra ci-dessous. Pour identifier des textes généalogiques foucaldiens, je me contenterai de tabler sur le fait relativement incontesté que Foucault s'est réclamé de la généalogie à l'époque où il enseignait au Collège de France. L'objectif de mon propos ne sera même pas de délimiter précisément ce corpus généalogique, mais de suggérer que l'anti-essentialisme de Nietzsche a joué un rôle matriciel pour les recherches de cette époque. J'en vois au moins deux indices textuels dans les cours au Collège de France : il s'agit de deux séquences réflexives où Foucault laisse entendre qu'il a fait sien cet anti-essentialisme, en revenant sur des travaux déjà effectués pour éclairer rétrospectivement la méthode qui a été suivie. La première de ces deux séquences figure dans Sécurité, territoire, population (leçon du 8 février 1978) et la deuxième ouvre quasiment Naissance de la biopolitique (leçon du 10 janvier 1979).

Je me reporte donc en premier lieu à Sécurité, territoire, population. Avant de prélever un passage précis de la leçon du 8 février 1978, il faut commencer par dire un mot sur son contexte. Foucault veut expliquer les raisons qui l'ont conduit à étudier l'histoire de la "gouvernementalité », en forgeant d'ailleurs ce néologisme. Il se fait à lui-même une objection : pourquoi ne pas avoir abordé plus directement le problème de l'État et de la population? C'est pour répondre à cette objection que Foucault revient sur le projet général qui a été le sien pendant plusieurs années, au sujet de l'armée, des hôpitaux, des écoles et des prisons. Foucault a appliqué à ces différents ensembles la problématique des disciplines. Il apporte maintenant une précision sur ses intentions : son objectif était chaque fois de "passer à l'extérieur », en trois sens différents qui sont distingués dans la suite de la leçon - passer à l'extérieur de l'institution, passer à l'extérieur de la fonction, et passer à l'extérieur de l'objet ${ }^{16}$. Le premier décentrement permettait de retrouver une technologie de pouvoir derrière l'institution, pour éviter l'«institutionnalocentrisme ». Le deuxième décentrement court-circuitait l'analyse purement fonctionnelle, pour adopter « le point de vue extérieur des stratégies et tactiques ${ }^{17}$. Et le troisième décentrement était une manière de « refuser de se donner un objet tout fait, que ce soit la maladie mentale, la délinquance, la sexualité $\rangle^{18}$. Voilà ce que Foucault dit lui-même de sa démarche dans cette rétrospection méthodologique. Or il me semble qu'on peut reconnaître en creux dans cette énumération la critique nietzschéenne des généalogies évolutionnistes. Ces généalogies partaient en effet des mots "bon et méchant», en se donnant donc un objet tout fait. Elles supposaient en outre des instincts ou des sentiments moraux, ce qui revient à hypostasier des entités psychologiques, comme on pourrait hypostasier des institutions sociales ou politiques. Enfin, l'évolution retracée dans ce cadre était orientée du début à la fin par une même fonction, ce que Nietzsche critiquait expressément comme une illusion finaliste. Foucault semble bien s'inscrire dans les pas de Nietzsche : on peut notamment comparer deux phrases, celle où Foucault dit que «l'histoire réelle de la prison n'est sans doute pas commandée par les succès et échecs de sa fonctionnalité », et celle du $\S 12$ du deuxième traité de la Généalogie de la morale, selon laquelle "le "but du droit" est bien la dernière chose dont il faille faire usage pour l'histoire de l'émergence du droit ». Dans ce $§ 12$, Nietzsche parlait d'ailleurs justement de l'histoire du droit pénal, sujet éminemment foucaldien. Et ce sous-texte renverrait bien sûr à la présence silencieuse de Nietzsche dans Surveiller et punir, même si je n'évoquerai pas ici ce long et fascinant chapitre ${ }^{19}$.

\footnotetext{
${ }^{15}$ Pour une caractérisation de la généalogie foucaldienne qui ne se veut pas pour autant une définition stricte, cf. REVEL (J.), Foucault, une pensée du discontinu, Paris, Mille et une nuits, 2010, en particulier p. 100-103.

${ }^{16}$ Cf. Foucault (M.), Sécurité, territoire, population. Cours au Collège de France (1977-1978), éd. établie par

M. Senellart, sous la direction de Fr. Ewald et A. Fontana, Paris, Seuil/Gallimard, 2004, p. 120.

${ }^{17}$ Ibid., p. 121.

${ }^{18}$ Ibid., p. 121-122.

19 À ce sujet, cf. SALANSKIS (E.), «"Une généalogie de 1'"âme" moderne” : sur l'inspiration nietzschéenne de Foucault dans Surveiller et punir », sous la direction de C. Denat, P. Wotling, Langage et pensée dans la philosophie française, Reims, Éditions et presses de l'université de Reims, 2016, p. 169-187.
} 
Je retiens seulement qu'une importante convergence méthodologique entre Nietzsche et Foucault se dessine déjà dans Sécurité, territoire, population. Or ce sentiment est conforté par une autre séquence réflexive, qui figure cette fois au début de Naissance de la biopolitique. Foucault commente de nouveau « un certain choix de méthode $»^{20}$, en tâchant cette fois d'expliquer, toujours à propos des pratiques gouvernementales, pourquoi il n'a pas cru bon d'invoquer de grandes notions politiques comme "le souverain, la souveraineté, le peuple, les sujets, l'État, la société civile » : Foucault désigne tous ces termes comme des " universaux ». Or il reproche justement à un certain historicisme de partir de ces universaux, pour étudier leurs variations historiques de manière seconde. L'inconvénient de cette méthode est de suggérer, délibérément ou non, qu'il existe des invariants sous-jacents aux transformations de l'histoire. Foucault souligne que son objectif est exactement inverse : " au lieu de partir des universaux pour en déduire des phénomènes concrets, ou plutôt que de partir des universaux comme grille d'intelligibilité obligatoire pour un certain nombre de pratiques concrètes, je voudrais partir de ces pratiques concrètes et passer en quelque sorte les universaux à la grille de ces pratiques ${ }^{21}$. On assiste donc chez Foucault à une suspension volontaire de la croyance aux universaux : supposons que ces universaux n'existent pas, et voyons quelle histoire on peut faire. Là encore, cette option méthodologique est bien celle pour laquelle la Généalogie de la morale avait plaidé. Au $\S 13$ du deuxième traité, Nietzsche observait précisément que « seul est définissable ce qui n'a pas d'histoire ». Par contraposition, cette phrase implique bien entendu aussi que ce qui a une histoire n'est pas définissable ${ }^{22}$. Nietzsche récusait donc comme pseudo-historiques toutes les généalogies qui partent d'une définition prétendument générale de leur objet. En guise d'illustration, il prenait l'exemple du châtiment, dont il recensait une douzaine de fonctions historiquement attestées, pour arriver à la conclusion que le châtiment échappe à toute définition : ce qui signifie que son supposé «concept» ne peut en aucun cas servir de fil conducteur généalogique. Nietzsche suggérait ainsi par avance l'idée que Paul Veyne défendra en 1978 dans «Foucault révolutionne l'histoire ": le généalogiste n'est pas à proprement parler relativiste, au sens où il prétendrait que les hommes ont perçu différemment le même objet à travers les siècles ${ }^{23}$. C'est justement cette supposée identité de l'objet qu'il faut remettre une bonne fois en question, en remarquant au contraire, comme le dit très bien Veyne, que « le point en question n'est pas le même d'une époque à l'autre $»^{24}$.

\section{La problématique des traces langagières}

Cette première partie négative pourrait laisser penser que le langage n'est décidément pas un bon fil conducteur généalogique. Mais une telle conclusion serait incomplète, car elle négligerait l'autre face de la médaille, c'est-à-dire le fait que la mise entre parenthèses des universaux ouvre la possibilité d'un traitement différent du langage. Effectivement, puisque les mots n'ont pas de signification idéale qui les arracherait en droit à l'histoire, leur histoire est elle-même significative. Elle est même, à certains égards, l'histoire précise qu'il s'agit de raconter, si on a à l'esprit une observation que Foucault fait avec raison dans L'Ordre du discours : « le discours n'est pas simplement ce qui traduit les luttes ou les systèmes de domination, mais ce pour quoi, ce par quoi on lutte, le pouvoir dont on cherche à s'emparer ${ }^{25}$. Même si Nietzsche n'est pas cité dans ce passage, cette affirmation de Foucault a incontestablement des allures nietzschéennes. En effet, Nietzsche signalait lui-même au $\S 2$ du premier traité de la Généalogie que dans certaines oppositions lexicales, l'instinct grégaire non seulement prend la parole, mais s'empare surtout des mots. En allemand, cette prise de pouvoir était signifiée par un jeu de mots ironique sur deux expressions proches, zu Worte kommen (« prendre la parole ») et $z u$ Worten kommen (« passer dans les mots ») ${ }^{26}$ : trait d'esprit qui suggérait

${ }^{20}$ Cf. Foucault (M.), Naissance de la biopolitique. Cours au Collège de France (1978-1979), éd. établie par M. Senellart, sous la direction de Fr. Ewald et A. Fontana, Paris, Seuil/Gallimard, 2004, p. 4.

${ }^{21}$ Ibid., p. 4-5.

${ }^{22}$ Il faut opposer réflexivement cette remarque aux tentatives de définition de la méthode généalogique qu'on trouve chez des auteurs comme Brian Lightbody : cf. LightBody (B.), Philosophical Genealogy. An Epistemological Reconstruction of Nietzsche and Foucault's Genealogical Method, New York, Peter Lang, 2010-2011, 2 vol.

${ }^{23}$ Cf. Veyne (P.), « Foucault révolutionne l'histoire » [1978], Comment on écrit l'histoire, Paris, Éditions du Seuil, 1996, p. 421.

${ }^{24}$ Ibid. De ce point de vue, toutes les relativisations sont encore tributaires d'un absolu implicite.

${ }^{25}$ Cf. Foucault (M.), L'Ordre du discours, Paris, Gallimard, 1971, p. 12.

${ }^{26} \mathrm{Cf}$. GM, I, § 2 : «c'est, pour me servir de mon langage, l'instinct grégaire qui finit par prendre la parole avec lui (et aussi par s'emparer des paroles) » (selon la traduction de Patrick Wotling). 
déjà que les mots sont une grande partie du pouvoir dont on cherche à s'emparer, conformément à l'interprétation nietzschéenne de l'origine du langage comme «extériorisation de puissance des dominants $»^{27}$.

Or s'il en est ainsi, on peut supposer que les mots gardent la trace des conflits ou des réappropriations dont ils ont successivement fait l'objet. Et ceci d'autant plus que les formes linguistiques apparaissent relativement stables par rapport aux différents sens qu'elles peuvent prendre au fil du temps dans différents contextes. Je fais ici allusion à une thèse célèbre du deuxième traité de la Généalogie de la morale: «La forme est fluide, mais le "sens" l'est plus encore... $\nu^{28}$. Dire cela, c'est suggérer que la forme est, certes, fluide, mais qu'elle l'est suffisamment moins que le sens pour garder la trace de mutations sémantiques qui lui sont advenues. Le pouvoir heuristique d'un mot apparait dès lors quand on le considère sous cet angle particulier, c'est-à-dire en tant que trace langagière.

Pour illustrer cette thèse, je voudrais donner deux exemples nietzschéens, que j'emprunte tous les deux à la Généalogie de la morale. Ils reposent à chaque fois sur des particularités de la langue allemande.

Mon premier exemple est la relation étymologique qui existe entre les mots schlecht et schlicht en allemand. Ces deux adjectifs sont devenus distincts dans la langue moderne, ce qui se traduit par le fait qu'ils ont chacun leur entrée de dictionnaire. On traduirait habituellement le premier par "mauvais» et le deuxième par "simple ». Mais d'un point de vue étymologique, ces deux mots n'en font qu'un, comme Nietzsche le souligne fortement au $\S 4$ du premier traité, et comme le confirmerait également le dictionnaire Grimm $^{29}$. La signification péjorative correspondant à schlecht semble d'ailleurs être apparue assez tardivement, par réinterprétation d'un emploi non péjoratif qui correspondrait aujourd'hui à schlicht. Nietzsche le relève et date plus précisément cette mutation de la guerre de Trente Ans, donc du XVII ${ }^{\mathrm{e}}$ siècle. Or ce petit fait apparemment anodin devient décisif pour l'ensemble du premier traité, en tant que trace généalogique d'un basculement des rapports de puissance exprimés dans le langage. Car il y a là une indication indirecte sur ceux qui ont fixé le sens des mots, avant et après la réinterprétation. Avant ce glissement, selon Nietzsche, " [schlecht] désignait originellement l'homme simple, l'homme commun, sans s'accompagner encore d'un regard de travers soupçonneux, simplement par opposition au noble ${ }^{30}$. Cet usage provenait donc d'une parole typiquement aristocratique. La désignation de "gens simples" s'inscrivait dans une logique nobiliaire d'autoglorification, qui n'exprimait qu'accessoirement ou secondairement un mépris du roturier, et d'ailleurs un mépris mêlé de compassion, si on suit jusqu'au bout l'analyse de Nietzsche. Mais avec la réinterprétation de schlecht, on assiste à ce que Foucault définirait comme un "événement» dans son article sur "Nietzsche, la généalogie, l'histoire » : c'est-à-dire "un rapport de forces qui s'inverse, un pouvoir confisqué, un vocabulaire repris et retourné contre ses utilisateurs $»^{31}$. Schlecht est tiré vers le sens de «mauvais», peut-être sous l'influence de böse, ce qui trahirait dans ce cas un retournement plébéien de l'usage aristocratique. On voit ici comment toute l'analyse du premier traité peut exploiter ce type de trace langagière.

Mais on peut en dire autant du deuxième traité, qui fournit à vrai dire une autre illustration, tout aussi importante, de la même approche. À partir du $\S 4$, pour étayer sa fameuse généalogie de la «mauvaise conscience », Nietzsche évoque tout particulièrement un fait de langue qui a, en effet, quelque chose de frappant en allemand: c'est la polysémie du mot «Schuld». On sait que ce terme a deux sens principaux, puisqu'il renvoie d'un côté au concept moral de «faute», de l'autre au concept matériel de " dette ». Pour Nietzsche, toute la question est d'expliquer comment un glissement a pu s'opérer du sens matériel au sens moral. Après s'être tourné assez longuement vers l'anthropologie du droit et de la religion, en exploitant probablement sa lecture de L'Origine de la civilisation de John Lubbock ${ }^{32}$, Nietzsche conclut que la mauvaise conscience est née d'une moralisation du concept de dette, rendue possible par l'idée d'une dette envers Dieu. Le christianisme serait l'aboutissement de ce processus, avec son Christ en croix qui

\footnotetext{
${ }^{27}$ Ibid.

${ }^{28}$ Ibid., II, § 12.

${ }^{29}$ Cf. Deutsches Wörterbuch von Jacob und Wilhelm Grimm, 16 Bde, in 32 Teilbänden, Leipzig 1854-1961, Leipzig, 1971, Online-Version vom 06.06.2018 : « die schlimme bedeutung [...], in der in neuerer sprache schlecht fast ausschlieszlich (abgesehen von der formel schlecht und recht) gebraucht wird, ist verhältnismäszig jung; die so entstehende zweideutigkeit des wortes war die veranlassung, dasz sich die nebenform schlicht im sprachgebrauche befestigte und die guten seiten der bedeutung von schlecht an sich zog ».

${ }^{30} \mathrm{Cf}$. GM, I, § 4.

${ }^{31}$ Cf. Foucault (M.), « Nietzsche, la généalogie, l’histoire », op. cit., p. 148.

${ }^{32}$ Cf. ThATCher (D.S.), « Nietzsche’s Debt to Lubbock », Journal of the History of Ideas, vol. 44, n 2, 1983, p. $293-$ 309.
} 
rachète nos fautes, comme si, précisément, nous avions à nous acquitter d'une dette envers un Dieu créancier $^{33}$. C'est un aspect essentiel de l'origine de la mauvaise conscience, qui prolonge l'hypothèse du $\S$ 16 au sujet de ses sources pulsionnelles. Or, de nouveau, Nietzsche suit un fil conducteur langagier, en traitant le mot Schuld comme une trace d'interprétations juridiques, religieuses et morales successives.

J'en arrive finalement à la question de savoir si cet aspect de la démarche de Nietzsche a été repris par Foucault. Et je voudrais suggérer brièvement que oui, en prenant un unique exemple, mais un exemple particulièrement célèbre et emblématique. C'est le travail de Foucault sur le mot grec $\pi \alpha \rho \rho \eta \sigma i ́ \alpha$. Dans le cours sur Le Gouvernement de soi et des autres, en particulier dans la leçon du 2 mars 1983, Foucault semble bien emprunter à son tour la « voie correcte » qui avait été définie par Nietzsche : il prend en effet pour fil conducteur de son investigation le mot grec $\pi \alpha \rho \rho \eta \sigma i ́ \alpha$. Il est vrai que Foucault parle aussi de la $\pi \alpha \rho \rho \eta \sigma i ́ \alpha$ comme d'une notion, ce qui pourrait par moments brouiller l'interprétation. Le début de la leçon du 2 mars dit exactement: «Le fil directeur que j'avais choisi pour le cours de cette année, c'était cette notion de parrêsia ${ }^{34}$. Mais il me semble que si on suit bien le mouvement de l'analyse, on s'aperçoit que Foucault ne présuppose pas, en réalité, qu'une unité notionnelle serait restée invariablement attachée à ce mot. Au contraire, il est attentif aux transformations que la $\pi \alpha \rho \rho \eta \sigma i ́ \alpha$ a subies, notamment dans la première moitié du $\mathrm{IV}^{\mathrm{e}}$ siècle av. J.-C., où il y a eu de son propre aveu « des modifications assez remarquables de cette notion de parrêsia ${ }^{35}$. Foucault résume ces modifications dans les paragraphes qui suivent. Initialement, la $\pi \alpha \rho \rho \eta \sigma i ́ \alpha$ était une notion démocratique, liée au contexte de la prise de parole devant l'assemblée du peuple : chez Thucydide, par exemple, Périclès use de sa $\pi \alpha \rho \rho \eta \sigma i ́ \alpha$ pour conseiller aux Athéniens de faire la guerre à Sparte $^{36}$. En l'occurrence, cette $\pi \alpha \rho \rho \eta \sigma i ́ \alpha$ était donc à l'époque la « liberté de parole» d'un citoyen qui, même dans le champ égalitaire de la démocratie, exerçait un ascendant sur les autres, parce qu'il était écouté, à ses risques et périls, quand il donnait publiquement son avis. Or, selon Foucault, cette notion initiale s'est transformée quand elle a été généralisée au-delà du contexte démocratique, pour être appliquée, en particulier, à la pratique du conseil au souverain. Sur ce nouveau terrain, la $\pi \alpha \rho \rho \eta \sigma i ́ \alpha$ a changé de cible, parce qu'elle ne visait plus simplement à conseiller sur les affaires de la cité, mais aussi à guider une âme individuelle, pour faire en sorte que cette âme se gouverne elle-même. C'est d'ailleurs ce " gouvernement de soi pour gouverner les autres » qui donne génériquement son titre au cours ${ }^{37}$. Quelle que soit la plausibilité de la reconstitution de Foucault, il paraît clair, sur le plan méthodologique, qu'on y retrouve les principaux aspects du fil conducteur langagier de Nietzsche : c'est-à-dire la stabilité relative d'un mot, des mutations du sens, et la possibilité d'utiliser le mot comme trace généalogique des mutations du sens. On pourrait même ajouter, en guise de cerise sur le gâteau nietzschéen, que Foucault étudie lui aussi un glissement axiologique et non pas simplement sémantique, puisqu'il évoque « le passage de la notion [de $\pi \alpha \rho \rho \eta \sigma i ́ \alpha$ à une certaine ambivalence, une certaine ambiguïté de valeur $»^{38}$. Montrer des fluctuations de valeur qui sont corrélatives de fluctuations du sens, c'est évidemment ce que Nietzsche avait entrepris de faire en 1887 en tant qu'historien des morales, des religions et du droit pénal.

\section{Conclusion}

J'ai essayé de montrer que Nietzsche et Foucault ont en commun une méthode de recherche qu'on peut qualifier de formelle, dans la mesure où elle tire de la forme relativement stable des mots un fil conducteur pour l'enquête historique. Même s'il est tentant d'identifier tout bonnement cette démarche à «la méthode généalogique », en toute rigueur, une telle assimilation serait inexacte : d'une part parce que Nietzsche critique aussi les « généalogistes de la morale jusqu'ici », ce qui veut dire qu'il existe de mauvaises pratiques généalogiques dont cette méthode formelle se distingue ${ }^{39}$; et d'autre part parce que définir la méthode

\footnotetext{
${ }^{33}$ Cf. GM, II, § 21.

${ }^{34}$ Cf. Foucault M., Le Gouvernement de soi et des autres. Cours au Collège de France (1982-1983), éd. établie par Fr. Gros, sous la direction de Fr. Ewald et A. Fontana, Paris, Seuil/Gallimard, 2008, p. 275.

${ }^{35}$ Ibid., p. 277.

${ }^{36}$ Ibid., p. 276.

${ }^{37}$ Ibid., p. 279.

${ }^{38}$ Ce qui serait dû au fait que la $\pi \alpha \rho \rho \eta \sigma i ́ \alpha$ est désormais menacée de dégénérer en flatterie : ibid., p. 277.

${ }^{39}$ L'idée que la généalogie serait intrinsèquement une bonne chose aux yeux de Nietzsche est en fait un contresens qui a été popularisé par Deleuze dans son livre sur Nietzsche et la philosophie. Dès la première section du livre, intitulée «Le concept de généalogie », Deleuze écrit en effet : « Nietzsche forme le concept nouveau de généalogie. Le philosophe est un généalogiste » (cf. DeleuZE (G.), Nietzsche et la philosophie, Paris, PUF, 1962, p. 2).
} 
généalogique absolument parlant pourrait bien être une manière de la déshistoriciser, c'est-à-dire de la mettre en contradiction avec une volonté de réhistorisation radicale que Nietzsche et Foucault ont partagée.

Il ressort en tout cas de cette présentation que Nietzsche et Foucault se sont opposés, l'un comme l'autre, à toute forme de platonisme dans le traitement de l'histoire. De ce point de vue, on ne saurait ériger Nietzsche en défenseur d'une conception platonicienne et supra-historique de la vérité qui viendrait contredire celle de Foucault, comme cela a été tenté récemment ${ }^{40}$. Je voudrais au contraire conclure cet article par une citation d'un beau fragment posthume de 1885, qui parle d'un « nous » dans lequel Nietzsche aurait certainement inclus Foucault : «Ce qui nous sépare le plus profondément de tous les modes de pensée platoniciens et leibniziens, c'est ceci : nous ne croyons pas aux concepts éternels, aux valeurs éternelles, aux formes éternelles, aux âmes éternelles ; et la philosophie, pour autant qu'elle est une science et non une législation, signifie seulement pour nous l'extension la plus grande du concept d'"histoire" »".

\footnotetext{
${ }^{40}$ Cf. Bouveresse (J.), Nietzsche contre Foucault. Sur la vérité, la connaissance et le pouvoir, Marseille, Agone, 2016, ainsi que la critique de cette critique qui est formulée par Daniele Lorenzini dans : LORENZINI (D.), La Force du vrai. De Foucault à Austin, Lormont, Le Bord de l'Eau, 2017, p. 6.

${ }^{41}$ Cf. en ligne, http://www.nietzschesource.org/\#eKGWB/NF-1885,38[14], trad. ES.
} 